\title{
Complicanze della nefrolitotrissia percutanea
}

\author{
A. Scavuzzo ${ }^{1}$, A. Granata ${ }^{2}$, A. Saita ${ }^{1}$, F. Fiorini ${ }^{3}$ \\ ${ }^{1}$ UOC Urologia, A.O.U. "Policlinico - Vittorio Emanuele", Catania \\ ${ }^{2}$ UOC Nefrologia e Dialisi, A.O.U. "Policlinico - Vittorio Emanuele”, Catania \\ ${ }^{3}$ UOC Nefrologia e Dialisi, Ospedale S. Maria della Misericordia, Rovigo
}

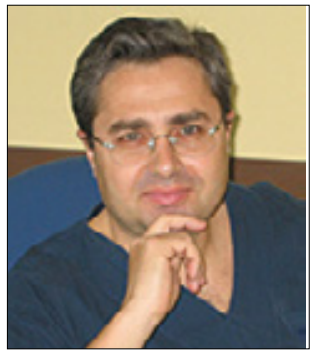

Alberto Saita

\section{Introduzione}

Negli ultimi 30 anni il trattamento dell'urolitiasi ha subito un notevole cambiamento, passando dalla chirurgia renale e ureterale a cielo aperto a tecniche mini-invasive, come la nefrolitotomia percutanea, l'ureteroscopia e la litotrissia con onde d'urto extracorporee (ESWL, Extracorporeal Shock Wave Lithotripsy), che offrono notevoli percentuali di successo in termini di accuratezza della rimozione dei calcoli con una significativa riduzione della morbilità e con notevole abbattimento dei costi. La nefrolitotrissia percutanea (PCNL) si inserisce in questo scenario di cambiamenti "miniinvasivi” per la prima volta nel 1976 (1), ma è solo nei primi anni Ottanta che ne viene dimostrata l'efficacia e la sicurezza nell'asportazione dei calcoli a stampo dalla pelvi renale (2). Negli ultimi anni, la PCNL ha acquistato grande autonomia e valenza terapeutica a seguito dei risultati e limiti dimostrati dalla ESWL (3), dell'impiego di strumenti flessibili e miniaturizzati per esplorare le vie escretici e dell'utilizzo di nuovi devices per la litotrissia (i.e. ultrasound/pneumatic devices, Holmium-YAGlaser) che hanno incrementato l'efficacia della litotomia sino a ottenere uno stone-free rate $>90 \%(4,5)$. Oggi la PCNL è considerata, secondo le linee guida della EAU (European Association of Urology) il gold standard nel trattamento della calcolosi a stampo e dei calcoli radiopachi (ossalato di Calcio) di dimensioni $>20 \mathrm{~mm}$ (Livello di Evidenza 1b; GR A), di cistina $>20 \mathrm{~mm}$ (LE 2; GR B). La PCNL rappresenta un'opzione terapeutica sicura con una bassa percentuale di complicanze: le più temibili possono verificarsi durante la puntura e comprendono le lesioni degli organi circostanti (colon, milza, fegato, pleura), mentre le altre complicanze specifiche postoperatorie sono rappresentate dalle emorragie e dalla febbre.

Nella presente review si descrive la procedura della PCNL step-by-step e si valutano le complicanze della nostra casistica, confrontandole con quelle riportate in letteratura.

\section{Materiali e Metodi}

Sono stati valutati retrospettivamente 390 pazienti (202

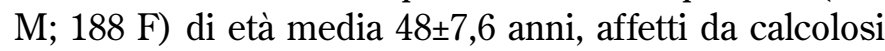
renale, sottoposti consecutivamente a PCNL presso la nostra U.O. a partire dall'aprile 2001 fino a settembre 2010; tra questi erano presenti 5 pazienti con rene a ferro di cavallo e 12 con diverticoli caliceali. Le procedure eseguite sono state 410 (390 PCNL e 20 second Look). Tutti i pazienti sono stati studiati con esame ecografico, urografico e TC al fine di definire sede e dimensioni del calcolo. La scintigrafia renale era effettuata nei casi di riduzione parenchimale all'ecografia o ritardo di eliminazione del mdc all'Uro-TC. Liter pre-operatorio comprendeva digiuno nelle 8 ore precedenti e antibioticoterapia profilattica parenterale o e.v (chinolonici, cefalosporine di III-IV generazione, aminoglicosidi). La procedura veniva effettuata in anestesia generale. Prima di iniziare il trattamento si introduceva per via retrograda un catetere ureterale (open-open 6-8Fr) fino alla giunzione pielo-ureterale per eseguire la pielografia ascendente pre-operatoria (Fig. 1). Il paziente era posto sul tavolo operatorio solitamente in decubito prono con le braccia ad angolo retto ai lati della testa, l'addome superiore e il torace di poco elevati rispetto al resto del corpo in modo 


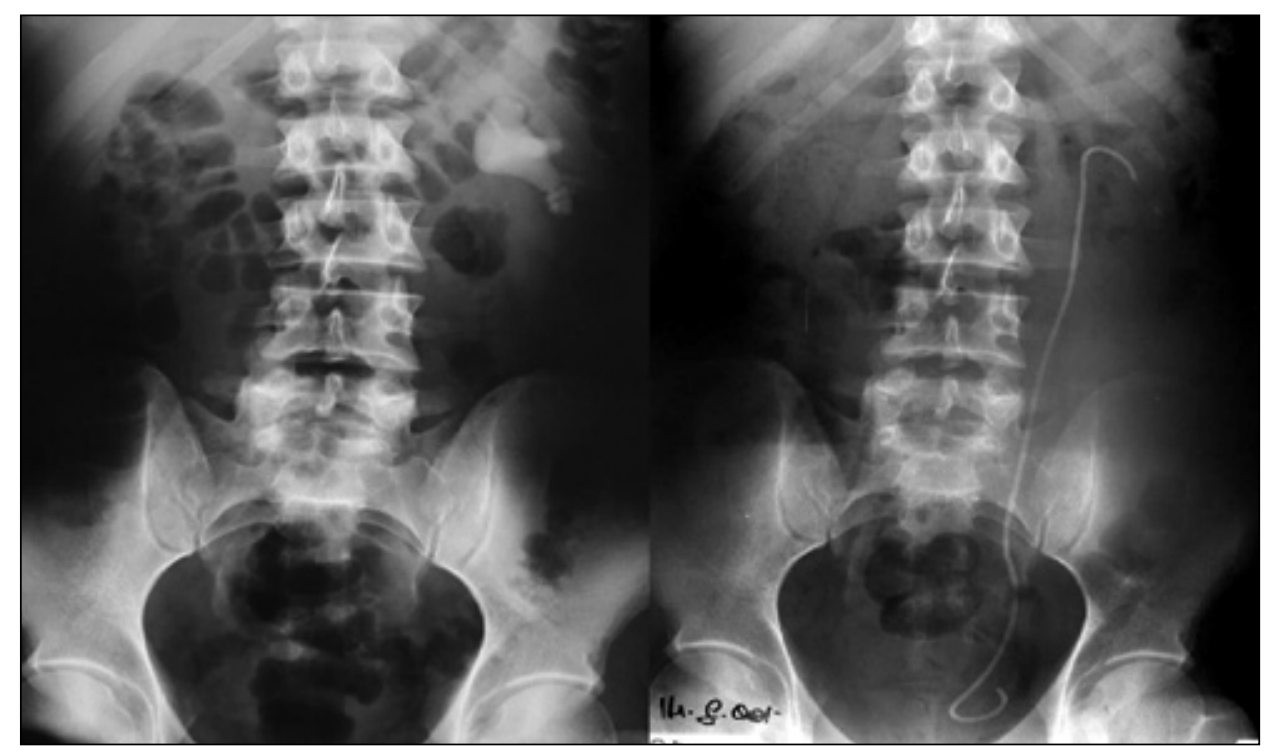

Fig. 1 - Prima di iniziare il trattamento si introduce per via retrograda un catetere ureterale (openopen 6-8Fr) fino alla giunzione pielo-ureterale per eseguire la pielografia ascendete pre-operatoria.

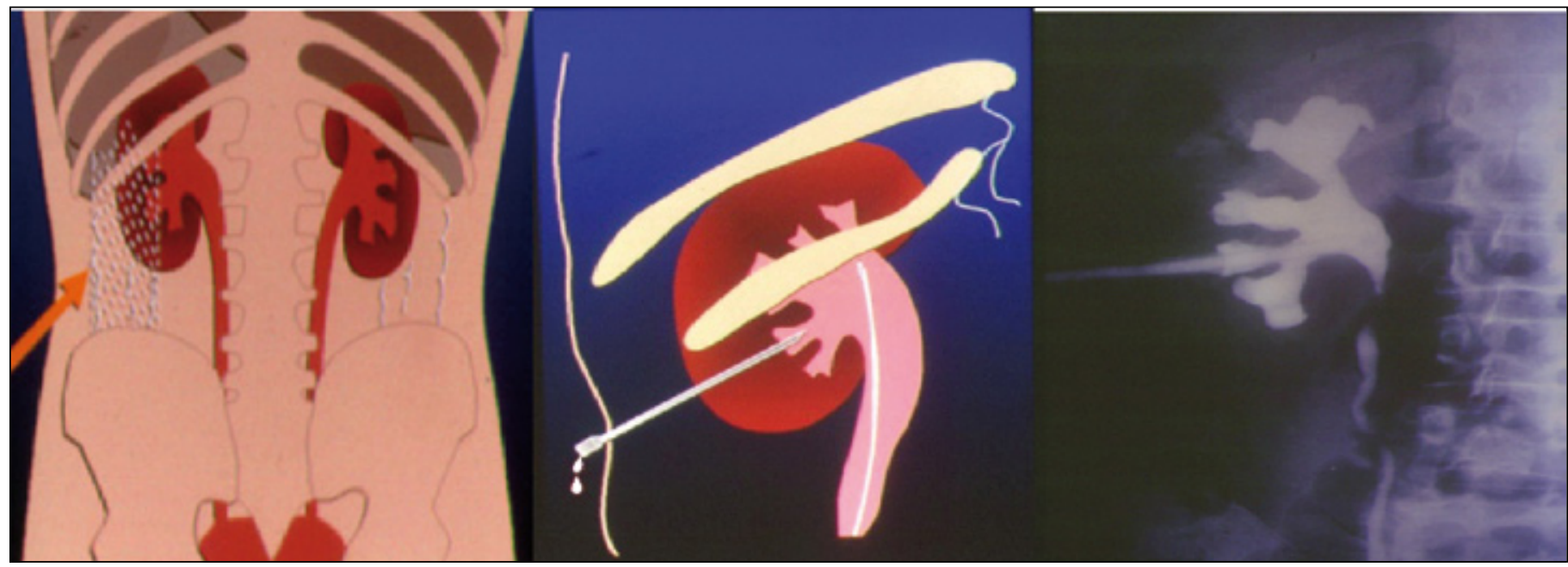

Fig. 2 - L'accesso al rene viene condotto per via transparenchimale, seguendo la stessa direzione del calice individuato: la zona di puntura da preferirsi è rappresentato dai calici inferiori e medi, così da attraversare l'unica porzione renale che solitamente non incrocia l'arteria segmentale maggiore, minimizzando il rischio di emorragia.

da creare una deflessione che aumentava la distanza fra $12^{\text {a }}$ costa e spina iliaca, e quindi l'area per un'adeguata puntura renale. L'accesso al rene, cruciale per il successo di ogni PCNL, veniva condotto per via transparenchimale, seguendo la stessa direzione del calice individuato: la zona di puntura da preferirsi è rappresentato dai calici inferiori e medi, così da attraversare l'unica porzione renale che solitamente non incrocia l'arteria segmentale maggiore, minimizzando il rischio di emorragia (Fig. 2). L'accesso al sistema collettore veniva realizzato tramite puntura radio-eco guidata del calice. Dopo la puntura del calice posteriore desiderato, si introduceva nel sistema collettore, attraverso l'ago, un filo guida "di sicurezza” (Super-stiff 0.035) e successivamente si creava "il canale di lavoro". Il tratto nefrostomico veniva dilatato mediante dilatatori in Teflon, dilatatori fasciali semirigidi tipo Amplatz in poliuretano, dilatatori telescopici o dilatatori a palloncino sotto controllo radiologico. Quindi si introduceva la camicia di Amplatz, della dimensione adeguata al tramite creato, con la quale ci si poteva facilmente orientare all'interno del sistema caliceale una volta introdotto il nefroscopio (Fig. 3). L'intera procedura, dall'introduzione dello strumento operativo in poi, veniva eseguita mantenendo l'irrigazione continua del tramite attraverso soluzioni composte da sodio cloruro allo $0,9 \%$ e acqua sterile. La frantumazione dei calcoli veniva eseguita con diversi sistemi di litotrissia: litotritore balistico (Lithoclast, 


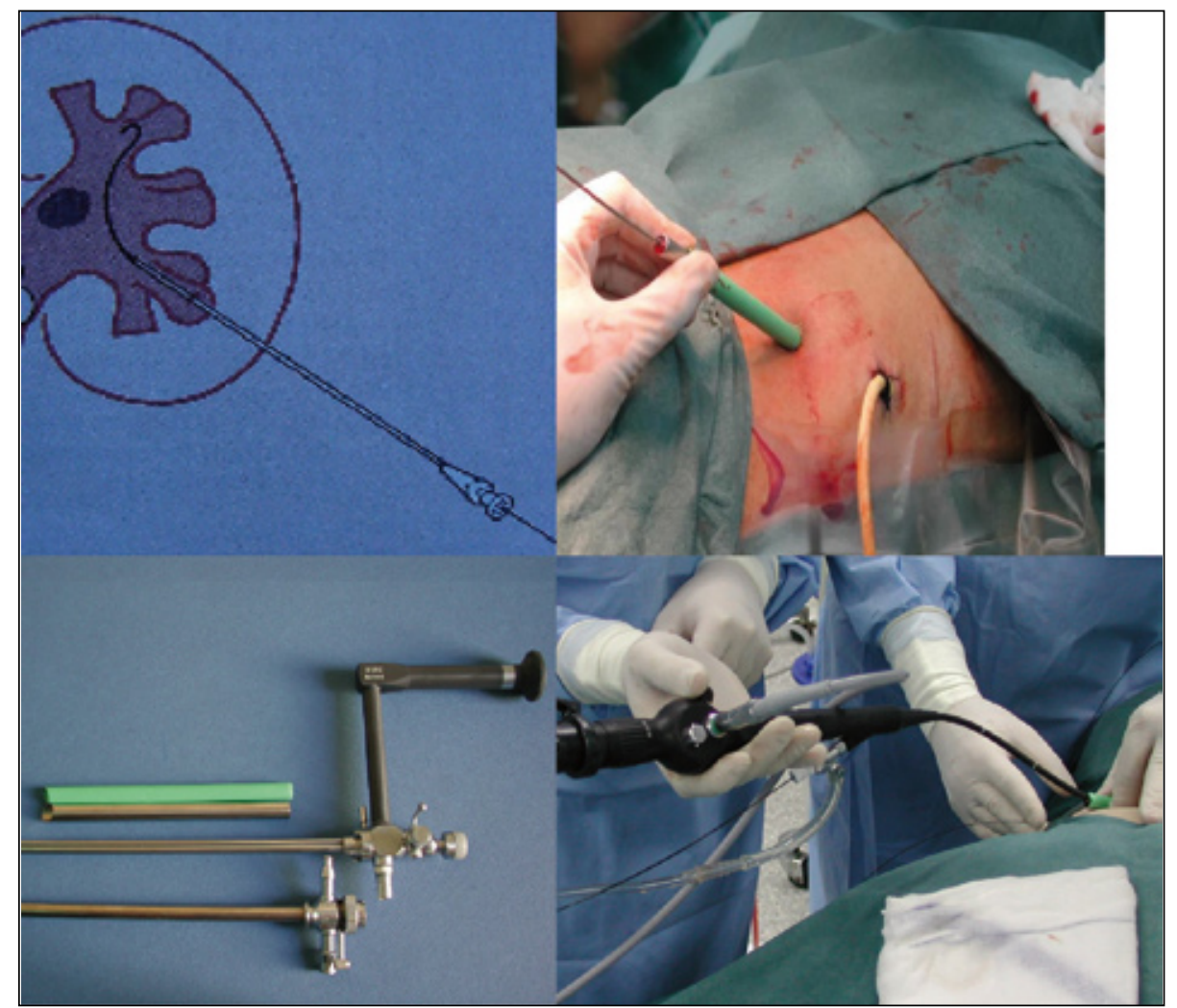

Fig. 3 - Il tratto nefrostomico viene dilatato mediante dilatatori in sotto controllo radiologico: si introduce quindi la camicia di Amplatz, della dimensione adeguata al tramite creato, così da poter facilmente orientare il nefroscopio all'interno del sistema caliceale.

Holmium-YAG-laser, litotritore a ultrasuoni). I frammenti ottenuti si asportavano con pinze o cestelli e si revisionavano le cavità escretici per assicurarsi del completo debulking della litiasi urinaria con nefroscopio flessibile. Alla fine della procedura si drenava il tramite nefrostomico con sonde tipo pigtail o cateteri Foley; il drenaggio nefrostomico veniva collocato attraverso la camicia di Amplatz, evitando di occludere il giunto pielo-ureterale. Dopo 24-48 ore dall'intervento, si effettuava la pielografia di controllo e il tubo nefrostomico veniva rimosso in assenza di stravasi e di calcoli residui.

\section{Risultati}

La percentuale di complicanze precoci verificatesi nella nostra casistica è stata del $26 \%$, molte delle quali risolte senza sequele (Tab. I). Le complicanze minori registrate sono state: episodi di iperpiressia (20\%), sanguinamenti clinicamente non significativi $(0,5 \%)$. Durante tutto lo studio sono stati osservati solo 2 casi $(0,5 \%)$ complicati da perdite emorragiche significative (riduzione media di $4 \mathrm{mg} / \mathrm{dL}$ di $\mathrm{Hb}$ ) nelle quali non c'è stata necessità di trasfusione. In uno di questi due casi l'ematuria si era verificata dopo 10 giorni dall'intervento ed era stata provocata da una fistola artero- venosa, evidenziata all'arteriografia renale, che è stata trattata con embolizzazione selettiva. Urosepsi $0,2 \%$ e infezioni delle vie urinarie (UTI) $2,5 \%$, trattate con antibiotico terapia. Si è avuto un caso di embolia polmonare $(0,2 \%)$ post-operatoria, che si è risolto con la terapia anticoagulante. La percentuale di stravaso

TABELLA I - PCNL ESEGUITA SU 390 PAZIENTI: COMPLICANZE

\begin{tabular}{lc}
\hline & CASISTICA (390 Pz) \\
\hline Stravaso & $6 \%$ \\
Emorragie renali & $0.5 \%$ \\
Trasfusioni & $0 \%$ \\
Fistole arterovenose & $0,2 \%$ \\
Febbre & $20 \%$ \\
Sepsi & $0,2 \%$ \\
Lesioni intestinali & $0 \%$ \\
Lesioni pleuriche & $0 \%$ \\
Pancreatiti acute & $0 \%$ \\
Mortalità perioperative & $0 \%$ \\
Embolia polmonare & $0,2 \%$ \\
\hline
\end{tabular}


TABELLA II - CONFRONTO TRA LE COMPLICANZE RIPORTATE IN LETTERATURA E LA NOSTRA CASISTICA

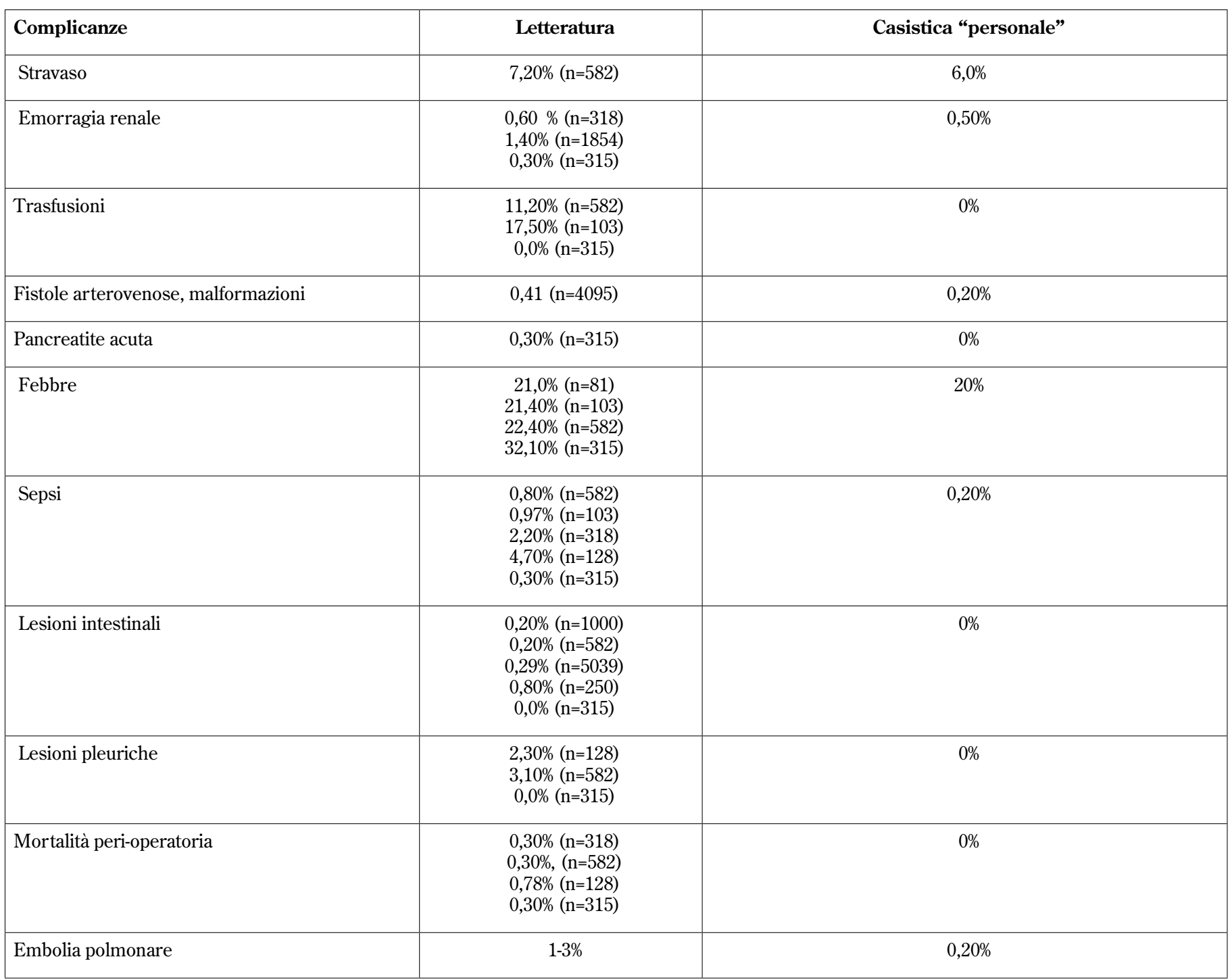

visibile radiologicamente è stata del $6 \%$, ma in nessun caso si è avuta la presenza di urinoma o di raccolte fluide richiedenti il drenaggio TC o eco-guidato.

\section{Review della letteratura}

In letteratura la percentuale totale di complicanze dopo PCNL varia ampiamente dal 29\% all' $83 \%$ (6-10). La percentuale di trasfusioni va dallo $0 \%$ al $17,5 \%(6,7$, 10); mentre la percentuale di sanguinamenti significativi è inferiore all'8\% (11). El-Nahas et al (12), hanno riportato su 4095 PCNL 41 casi $(0,1 \%)$ di emorragie significative risoltesi con embolizzazione selettiva, mentre altri autori $(13,14)$ hanno riportato una incidenza di urosepsi dello 0,9-4,7\%. Le percentuali di complicanze legate all'accesso percutaneo, riportate in letteratura $(15,16)$, sono $2,3-3,1 \%$ per le lesioni pleuriche e $0,2-0,8 \%$ per le lesioni intestinali. In Tabella II sono confrontati i dati di questo lavoro con quelli della letteratura.

\section{Discussione}

Le complicanze della PCNL possono essere divise in complicanze correlate all'accesso e correlate alla rimozione dei calcoli (7). Le complicanze sono essenzialmente attribuibili a una inadeguata selezione dei pazienti, alla mancanza di un adeguato equipment e a errori di tecnica (7). Una corretta selezione dei pazienti è particolarmente importante per la PCNL. La PCNL è controin- 
dicata nei pazienti affetti da coagulopatie, da gravi infezioni delle vie urinarie o da pionefrosi. La presenza di malattie concomitanti come il diabete mellito, le malattie polmonari, cardiovascolari, disfunzioni renali o la calcolosi infetta aumentano il rischio di risultati sub-ottimali. La procedura diviene difficoltosa in caso di obesità, malformazioni della colonna vertebrale, pelvi bifida, rene a ferro di cavallo o malrotazione del rene. La complicanza più temuta correlata all'accesso è il sanguinamento parenchimale, che può verificarsi durante la procedura e può impedirne il completamento. Nei casi di sanguinamento persistente e rilevante, è necessario effettuare un'angiografia renale e valutare la possibilità di una embolizzazione. Sanguinamenti tardivi (3 settimane dopo la procedura) possono essere provocati da fistole arterovenose, da pseudoaneurismi o da malformazioni artero-venose $(11,12,17,18)$. Durante l'accesso vi è il rischio di provocare lesioni pleuriche, duodenali, intestinali e agli altri organi, che possono essere scongiurate effettuando un imaging completo pre-operatorio e realizzando la puntura del rene eco-guidata e radio-guidata (19). Ciò consente un approccio preciso all'organo, in quanto permette di stabilire l'asse dei calici e di direzionare l'ago in maniera precisa verso il calice desiderato. $\grave{E}$ anche possibile pungere il rene con l'ausilio della sonda ecografica; tale tecnica detta "free-hand puncture" viene realizzata solo con una sonda ecografica settoriale e richiede grande esperienza. Le complicanze correlate alla rimozione dei calcoli sono: la setticemia, lo stravaso, il riassorbimento di fluidi da irrigazione e la "steinstrasse”. Il rischio di setticemia aumenta nei casi di calcolosi infetta, insufficienza renale, disseminazione di infezioni dal tramite cutaneo. Nei casi di pionefrosi bisogna soprassedere al trattamento endoscopico, somministrare terapia antibiotica adeguata e drenare il rene (20). La durata dell'intervento e l'eccesso di quantità di liquido di irrigazione possono essere causa di sepsi (21). E importante, per prevenire le complicanze settiche, mantenere basse pressioni durante l'irrigazione del sistema e ridurre i tempi operatori (22), somministrare antibiotici prima dell'intervento, anche se le urine sono sterili in fase pre-operatoria (23). Gli effetti della PCNL sul parenchima renale e sulla funzionalità renale sono minimi (24-26), ma i pazienti affetti da calcolosi a stampo e complessa hanno un rischio maggiore di sviluppare un deterioramento della funzionalità renale (28).

\section{Conclusioni}

La PCNL è un trattamento sicuro e associato a un basso tasso di complicanze. Per evitare le temute complicanze e assicurarsi un outcome ottimale, prima dell' intervento occorre pianificare e considerare diversi fattori:

- Adeguata valutazione pre-operatoria

- Adeguata puntura del calice desiderato

- Puntura eco e radio-guidata

- Atraumatica dilatazione sotto controllo radiologico

- Minime angolazioni dello strumento

- Uso dello strumento flessibile per i calcoli del tratto superiore

- Riconoscere quando fermarsi e come trattare le complicanze.

Una buona conoscenza della tecnica e del follow-up postoperatorio sono essenziali per ridurre e gestire eventuali complicanze.

\section{Riassunto}

L'obiettivo di questo studio è descrivere "step-by-step" la nefrolitotrissia percutanea e le sue complicanze. A tale proposito sono stati valutati i casi di calcolosi renale da noi trattati con nefrolitotrissia percutanea (PCNL) dal $2001 \mathrm{al} 2010$ ed è stata al contempo rivista la letteratura circa l'incidenza e il management delle complicanze legate a tale procedura. La percentuale globale di complicanze registrate durante e dopo le PCNL possono raggiungere anche l' $83 \%$ dei casi e comprendono ematomi $(7,2 \%)$, trasfusioni $(11,2 \%-17,5 \%)$ e febbre $(21,0 \%-32,0 \%)$. Molte di queste complicazioni, se riconosciute precocemente, possono essere gestite con trattamenti conservativi o mininvasivi. Le complicanze maggiori sono rare (setticemia $(0,3-0,4 \%)$, lesioni intestinali $(0,2-0,8 \%)$, lesioni pleuriche $(0,0-3,1 \%))$. Le co-morbilità (insufficienza renale, diabete mellito, obesità severa, malattie polmonari) sembrano incrementare il rischio di complicanze. Nella nostra casistica la percentuale complessiva di complicanze è stata pari al $26 \%$, molte delle quali si sono risolte senza sequele. In conclusione, la PCNL rappresenta una metodica sicura e con basso rischio di complicanze specifiche specie se a seguito di un'accurata selezione e preparazione dei pazienti, tecnica appropriata e attento follow-up nel post-operatorio al fine di assicurare tempestivo intervento se/quando necessario.

Parole chiave: Nefrolitotrissia percutanea, Complicanze, Litiasi renale

Indirizzo degli Autori:

Antonio Granata, MD

Via F. Paradiso 78/a

95024 Acireale (CT)

antonio.granata4@tin.it 


\section{Bibliografia}

1. Fernstrom I, Johansson B. Percutaneous pyelolithotomy: a new extraction technique. Scandinavian Journal of Urology and Nephrology 1976; 10: 257.

2. Alken P, Hutschenreiter G, Gunther R, Marberger M. Percutaneous stone manipulation. J Urol 1981; 125: 463-6.

3. Rassweiler JJ, Renner C, Chaussy C, Thüroff S. Treatment of renal stones by extracorporeal shock wavelithotripsy. Eur Urol 2001: 40: 54-64.

4. Hafron J, Fogarty JD, Boczko D, Hoenig DM. Combined ureterorenoscopy and shockwave lithotripsy for large renal stone burden: an alternative to percutaneous nephrolithotomy? J Endourol 2005; 19: 464-8.

5. Marguet CG, Springhart WP, Tan YH, et al. Simultaneous combined use of flexible ureteroscopy and percutaneous nephrolithotomy to reduce the number of access tracts in the management of complex renal calculi. BJU Int 2005; 96: 1097-100.

6. Osman M, Wendt-Nordahl G, Heger K, Michel MS, Alken P, Knoll T. Percutaneous nephrolithotomy with ultrasonography-guided renal access: experience from over $300 \mathrm{ca}-$ ses. BJU Int 2005; 96: 875-8.

7. Michel M.S, Trojan L.,Rassweiler J.J. Complication percutaneous nephrolithotomy. Eur Urol 2007; 51: 899-906.

8. Tefekli A, Ali Karadag M, Tepeler K, et al. Classification of percutaneous nephrolithotomy complications using the modified clavien grading system: Looking for a standard. Eur Urol 2008; 53: 184-90.

9. Skolarikos A, de la Rosette J. Prevention and treatment of complications following percutaneous nephrolithotomy. Curr Opin Urol 2008; 18: 229-34.

10. de la Rosette J, Assimos D, Desai M, Gutierrez J, Lingeman J, Scarpa R, Tefekli A, on behalf of the CROES PCNL Study Group. The Clinical Research Office of the Endourological Society Percutaneous Nephrolithotomy Global Study: Indications, Complications, and Outcomes in 5803 Patients. J Endourol 2011, 25: 11-6.

11. Srivastava A, Singh KJ, Suri A. Vascular complications after percutaneous nephrolithotomy: are there any predictive factors? Urology 2005; 66: 38-40.

12. el-Nahas AR, Shokeir AA, Mohsen T, Gad H, el-Assmy AM, el-Diasty T, el-Kappany HA. Functional and morphological effects of postpercutaneous nephrolithotomy superselective renal angiographic embolization. Urology 2008; 71(3): 408-12.

13. Aron M, Yadav R, Goel R, et al. Multi-tract percutaneous nephrolithotomy for large complete staghorn calculi. Urol Int 2005; 75: 327-32.

14. Vorrakitpokatorn P, Permtongchuchai K, Raksamani EO, Phettongkam A. Perioperative complications and risk fac- tors of percutaneous nephrolithotomy. J Med Assoc Thai 2006; 89: 826-33.

15. Netto Jr NR, Ikonomodis J, Ikari O, Claro JA. Comparative study of percutaneous access for staghorn calculi. Urology 2005; 65: 659-63.

16. Negrete-Pulido OR, Molina-Torres M, Gutiérrez-Aceves J. Colonic perforation during percutaneous nephrolithotomy: prevention, diagnosis and treatment. Rev Mex Urol 2010; 70(1): 44-7.

17. Clayman RV, Surya V, Hunter D, et al. Renal vascular complications associated with the percutaneous removal of renal calculi. J Urol 1984; 132: 228-30.

18. Rastinehad A, Andonian S, Smith A, Siegel D. Management of hemorrhagic complications associated with percutaneous nephrolithotomy. J Endourol 2009; 23(10): 1-5.

19. Watterson JD, Soon S, Jana K. Access related complications during percutaneous nephrolithotomy: urology versus radiology at a single academic institution. J Urol 2006; 176: 142-5.

20. Dogan H.S., Sahin A, Cetinkaya Y, Akdogan B, Ozden E, Kendi S. Antibiotic prophylaxis in percutaneous nephrolithotomy: prospective study in 81 patients. J Endourol 2002; 16: 649-53.

21. Preminger GM, Assimos DG, Lingeman JE, Nakada SY, Pearle MS, Wolf JS. Chapter 1: AUA guideline on management of staghorn calculi: diagnosis and treatment recommendations. J Urol 2005; 173: 1991-2000.

22. Takeuchi H, Ueda M, Nonomura M, et al. [Fever attack inpercutaneous nephrolithotomy and transurethral ureterolithotripsy]. Hinyokika Kiyo 1987; 33: 1357-63.

23. Dogan HS, Sahin A, Cetinkaya Y, Akdogan B, Ozden E, Kendi S. Antibiotic prophylaxis in percutaneous nephrolithotomy: prospective study in 81 patients. J Endourol 2002; 16: 649-53.

24. Lechevallier E, Siles E, Ortega JC, et al. Comparison by SPECT of renal scars after extracorporeal shock wave lithotripsy and percutaneous nephrolithotomy. J Endourol 1993; 7: 465.

25. Urivetsky M, Motola J, King L, et al. Impact of percutaneous renal stone removal on renal function: assessment by urinary lysozyme activity. Urology 1989; 33: 305-8.

26. Chatham JR, Dykes TE, Kennon WG, et al. Effect of percutaneous nephrolithotomy on differential renal function as measured by mercaptoacetyltriglycine nuclear renography. Urology 2002; 59: 522-6.

27. Teichman JM, Long RD, Hulbert JC. Long-term renal fate and prognosis after staghorn calculus management. J Urol 1995; 153: 1403-7.

28. Samir ST, Complication of urology surgery. Complication of percutaneous surgery. 2010, Chapter 27: 305-17. 